\title{
Wideband radar detector based on characteristic parameters of echoes
}

\author{
CHANG Jiayun, FU Xiongjun*, JIANG Wen, and XIE Min
}

School of Information and Electronics, Beijing Institute of Technology, Beijing 100081, China

\begin{abstract}
The statistical characterization of radar range cells with the target signals is much more distinct than that of the range cells with noise-only signals. Hence, the quasi-optimal detection principle based on the characteristic parameters of echo signals is adopted to develop a detector of range-spread targets in Gaussian noise. Firstly, the characteristic parameters of the return signals in the entire range profiles of radar are investigated. Secondly, the clustering analysis of the characteristic parameter matrix is discussed to extract the test statistic of echoes. Finally, the probabilities of detection and false alarm of the proposed detector are provided. Theoretical analysis shows that the proposed detector does not need the prior knowledge about the spatial distribution of the target scattering centers in practical scenarios, and it is simple and robust even in low signal-to-noise ratio (low-SNR) scenarios. Monte Carlo (MC) simulations reveal that the detection performance of the proposed detector outperforms the conventional detectors.
\end{abstract}

Keywords: wideband radar, mathematical statistics, characteristic parameter, clustering analysis.

DOI: $10.21629 / J S E E .2019 .05 .08$

\section{Introduction}

The point-target model for narrowband radar may fall in many practical applications, such as the detection of aircraft or ships, composed of multiple physical scatterings $[1,2]$. For high resolution radars (HRRs) [3-5], targets are spatially divided into a number of scattering centers along the high resolution range profile (HRRP) [6-8]. In this case, compared with conventional narrowband radars, the received signals of HRRs contain more abundant targets and environment information. Besides, due to the increasing of the radar range resolution, the energy per cell backscattered by the clutter will be reduced. Furthermore, the fluctuation of scattering centers is less than point-like targets in narrowband low-resolution radars (LRRs) [9],

\footnotetext{
Manuscript received January 24, 2018.

*Corresponding author.

This work was supported by the National Natural Science Foundation of China (61571043) and the 111 Project of China (B14010).
}

which contributes to obtaining important enhancement of the detection performance. Thus, HRRs have widely been applied for target imaging, recognition, and classification $[10,11]$. Nowadays, especially, to detect range-spread targets and then realize the integration of detection, recognition and classification have received close and extensive attention [12-17].

To detect a range-spread target, intensive researches have been developed during the past decades. In [18], a stochastic knowledge-aided (KA) model was introduced for detecting of range-spread targets in distributed multiple-input multiple-output (MIMO) radar [19,20], but the strict orthogonality was not taken into consideration. In [21,22], an adaptive detection method of range-spread targets in Gaussian noise was derived under the assumption that the noise shares the same block-diagonal covariance structure. In [23], a detector was proposed to detect range-spread aircraft in white Gaussian noise, and the detector can work well even in the mild condition without a strict target mode. However, the influence of the rotational motion of targets is not taken into account in the aforementioned detector. In [24], exploiting the coherent integration method, a developed detector in Gaussian noise was given. In [25], a new range-spread target detector for missileborne radar in white Gaussian clutter was provided. However, these two detectors cannot be adapted into the detection of targets with high velocity. In [26], an adaptive detector of a range distributed target in Gaussian interference was devised under the assumption that some information about noise components was pre-determined. In [27], based on the waveform entropy of the arithmetic average, a developed detector in white Gaussian noise was presented. In [28], under a wide assumption of target echoes, an efficient detector of air targets in white Gaussian noise background is derived. However, the aforementioned detectors are only suitable for detecting targets of low rotational velocity. The problem of detecting a moving range-spread target in distributed MIMO radar was solved in [29] under 
the assumption that the target echoes were known. Based on the persymmetric structures in return signals, the detection problem of the distributed MIMO radar was discussed [30]. In [31], developed within the generalized likelihood ratio test (GLRT) [32-35] framework, the two detectors in distributed MIMO with moving platforms were derived. However, work in [30,31] assumed that the covariance matrices for all transmit-receive (Tx-Rx) pairs of MIMO radar were the same.

In this paper, firstly, quasi-optimal detection criteria based on the echo characteristic parameters are incorporated in the detector design procedure for the distributed target detection problem in white Gaussian noise. A detector for broadband radar based on characteristic parameters of echoes is proposed. Secondly, the probability of false alarm and the detection probability are provided. Finally, theoretical analysis shows that the proposed detector is non-dependent on the prior distribution information of target scattering centers. Monte Carlo (MC) simulations illustrate that the proposed detector outperforms the conventional detector.

The rest of this paper is organized as follows. In Section 2 , the proposed detector based on characteristic parameters of echoes is derived and discussed. Then, the effectiveness of the proposed detector is verified by MC simulations in Section 3. Finally, conclusions are given in Section 4.

\section{Proposed detector}

Radar signals are random, and all observed signals are uncertain variables which are fluctuating randomly depending on the space and the time. For all these randomly fluctuating variables, it is effective to utilize the mathematical statistic method to describe their statistical characteristics. The statistic characteristics of the target echo are different from that of the noise-only signals. For example, the observed Gaussian white noise signals are zero-mean data with variance 1 , the mean value of target signals changes from 0 to 1 in terms of the mathematical statistic characteristics.

Based on the characteristic parameters of echo, calculate the coefficient matrix of polynomial fitting curve $\boldsymbol{W}$

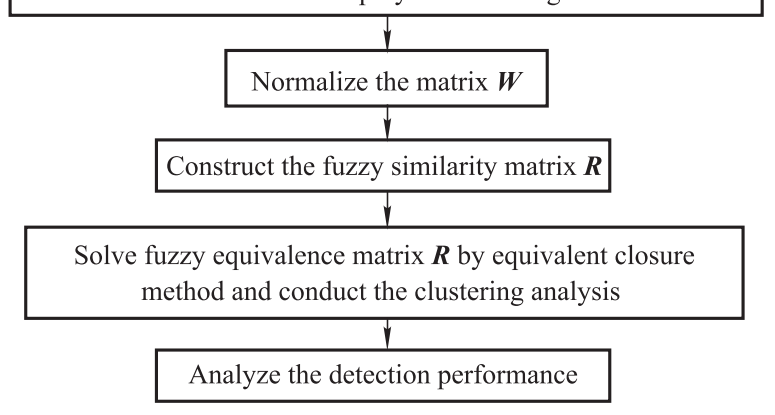

Fig. 1 Scheme flowchart of the proposed detector
Therefore, the proposed detector is derived depending on different mathematical statistic characteristics between target echoes and noise-only echoes. The scheme flowchart of the proposed detector is shown in Fig. 1.

\subsection{Characteristic parameters of echoes}

In this paper, the characteristic parameters of return signals derive from the coefficient matrix of the polynomial fitting curve, and the curve refers to a histogram statistical curve of data collected from range cells. When there is no target echoes in the sampling gate, in other word, all data in the range cells only contain noise and/or clutter, characteristic distribution curves of data in those range cells are stable and similar. However, when the return signals contain target echoes, statistic characteristics of target echoes will change dramatically. Hence, the statistical histograms of two types of target echoes and noise-only echoes represent completely different shapes. In this case, their polynomial fitting coefficient matrices obtained from histogram statistical curves are quite different from each other. Hence, based on the mathematical statistic characteristics of return signals, we can effectively accomplish the detection of range-spread targets.

Based on the characteristic parameters of echo mentioned above, we formulate the distributed target detection during the entire observation scenario to confirm the following discussion. Suppose that the detection background is Gaussian white noise, and the radar transmits the linear frequency modulation (LFM) [36] signal

$$
s_{s}=A \cdot \operatorname{rect}\left(\frac{\tau}{T_{p}}\right) \cdot \mathrm{e}^{\mathrm{j} 2 \pi \cdot \frac{1}{2} k t^{2}}
$$

where $\tau$ is the pulse duration, $k=\frac{B}{\tau}$ is the frequency modulation (FM) slope, $B$ is the bandwidth, $T_{p}$ is the pulse recurrence time, and $A$ is the signal amplitude. In this paper, the problem of interest is to detect a range-distributed target in Gaussian white noise. The data is collected from $N$ range cells. Thus, the detection problem can be formulated in terms of the following binary hypothesis test [37]:

$$
\left\{\begin{array}{l}
\boldsymbol{H}_{0}: \boldsymbol{x}=\boldsymbol{w} \\
\boldsymbol{H}_{1}: \boldsymbol{x}=\boldsymbol{s}+\boldsymbol{w}
\end{array}\right.
$$

where $s=\left\{s_{1}, s_{2}, \ldots, s_{N}\right\}$ and $\boldsymbol{w}=\left\{w_{1}, w_{2}, \ldots, w_{N}\right\}$ are the target signals and noise-only signals after matching filtering, respectively, and $N$ denotes the number of range cells. The noise is assumed to be zero-mean complex white Gaussian noise with variance equal to $\sigma^{2}$. Other related parameters of the signal model in the simulation are listed in Table 1 . 
Table 1 Simulation parameters of signal model

\begin{tabular}{ccc}
\hline Parameter & Name & Value \\
\hline \multirow{4}{*}{ Radar } & Signal amplitude/V & 1 \\
parameter & Carrier frequency $/ \mathrm{GHz}$ & 10 \\
& Bandwidth/MHz & 500 \\
& Pulse duration $/ \mu \mathrm{s}$ & 10 \\
& Pulse repetition frequency $(\mathrm{PRF} / \mathrm{Hz}$ & 200 \\
\hline \multirow{3}{*}{ Target } & Original distance to $\mathrm{radar} / \mathrm{m}$ & 5000 \\
parameter & Radial velocity $/ \mathrm{m} \cdot \mathrm{s}^{-1}$ & 0 \\
& Target radial length $/ \mathrm{m}$ & 30 \\
& Number of scatters & 10 \\
\hline \multirow{3}{*}{ Sampling } & Width of range gate $/ \mu \mathrm{s}$ & 30.2 \\
parameter & Ending time $/ \mu \mathrm{s}$ & 58.53 \\
& Starting time $/ \mu \mathrm{s}$ & 28.33 \\
& Sampling rate $/ \mathrm{MHz}$ & 625 \\
\hline
\end{tabular}

Note that whether the target is moving or not in the mono-pulse radar system, it does not affect the detection performance of the proposed detector; relative distribution positions of scattering centers, that is, the normalized distances between the position of scattering centers and the front part of the target are $0.01,0.1,0.15,0.3,0.31,0.7$, $0.71,0.72,0.9,0.91$. The amplitudes of scattering points are $20,30,30,30,20,20,20,50,40,20$, respectively.

Assume that average false alarm time $T_{\mathrm{fa}}$ is $50 \mathrm{~s}$, then the false alarm probability $P_{\mathrm{fa}}$ can be calculated as

$$
P_{\mathrm{fa}}=\frac{\mathrm{PRT}}{T_{\mathrm{fa}}}=\frac{1}{\mathrm{PRF} \cdot T_{\mathrm{fa}}}=\frac{1}{200 \times 50}=1 \times 10^{-4} .
$$

Suppose that the minimum radial length of the target is $20 \mathrm{~m}$, and the maximum is $40 \mathrm{~m}$, the gain of the match filter is 1 , and the length of the sliding window is 20 times of the minimum radial length of the target. The step size of the sliding window is a quarter of the radial length of the target. The partition number of the statistical histogram is 20 , and it is curved by the 4th-degree polynomial fitting coefficient.

\subsubsection{Statistical characteristics of noises-only echoes}

The statistical histogram of data in all the sliding windows with noises only and the corresponding 4th-order polynomial fitting curves are shown in Fig. 2 and Fig. 3.

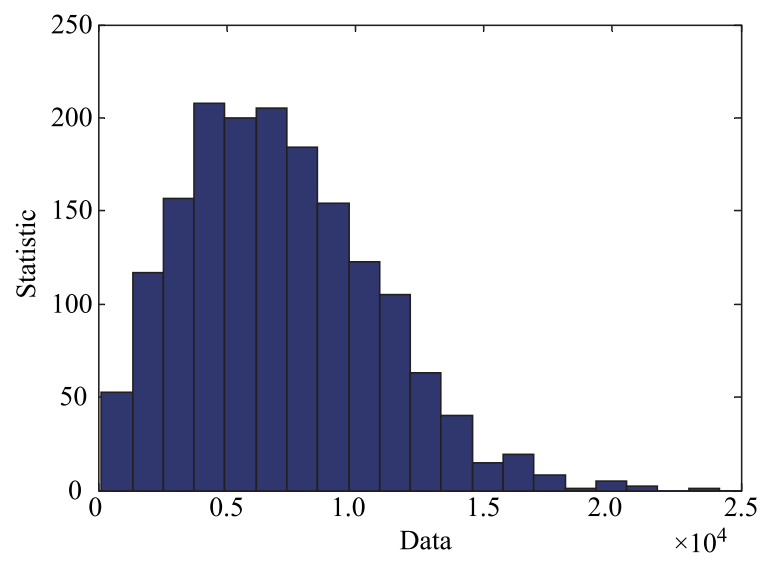

Fig. 2 Statistical histogram of noise-only echoes

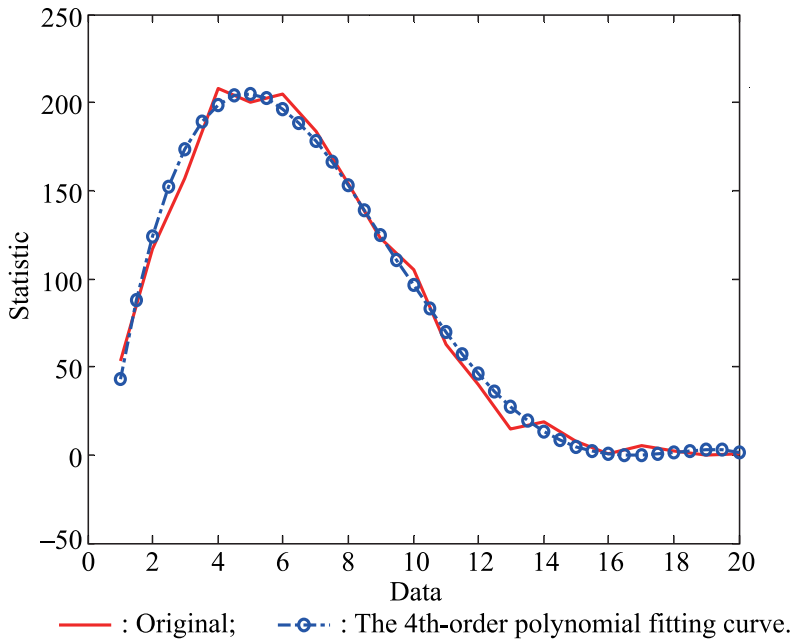

Fig. 3 Fitting curve of noise-only echoes

\subsubsection{Statistical characteristics of target echoes}

Two cases, where the signal-to-noise ratio (SNR) is $25 \mathrm{~dB}$ and $15 \mathrm{~dB}$ respectively, are taken for example to illustrate the statistical characteristics of target echoes. The results show that, the statistical histogram of target echoes is obviously different from that of noise-only echoes, as shown from Fig. 4 to Fig. 7.

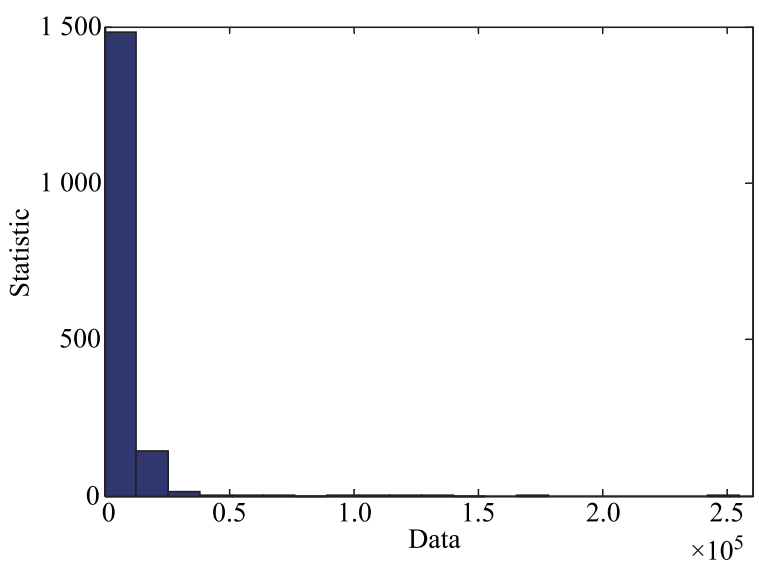

Fig. 4 Statistical histogram of target echoes $(\mathrm{SNR}=25 \mathrm{~dB})$

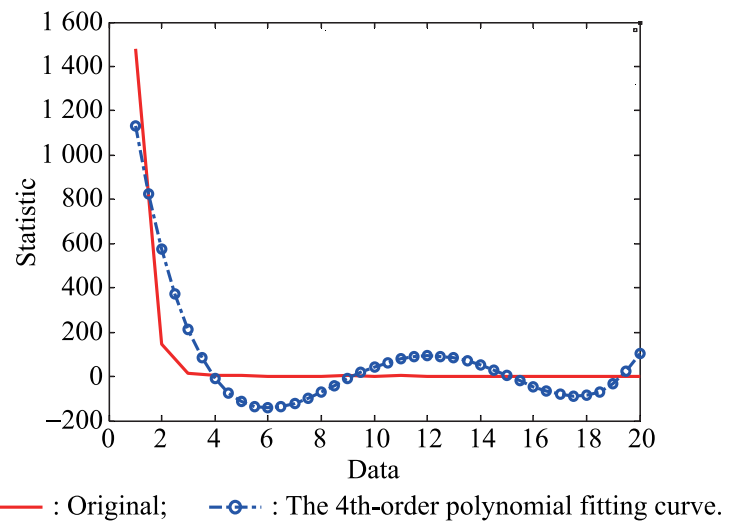

Fig. 5 Polynomial fitting curve of target echoes $($ SNR $=25 \mathrm{~dB})$ 


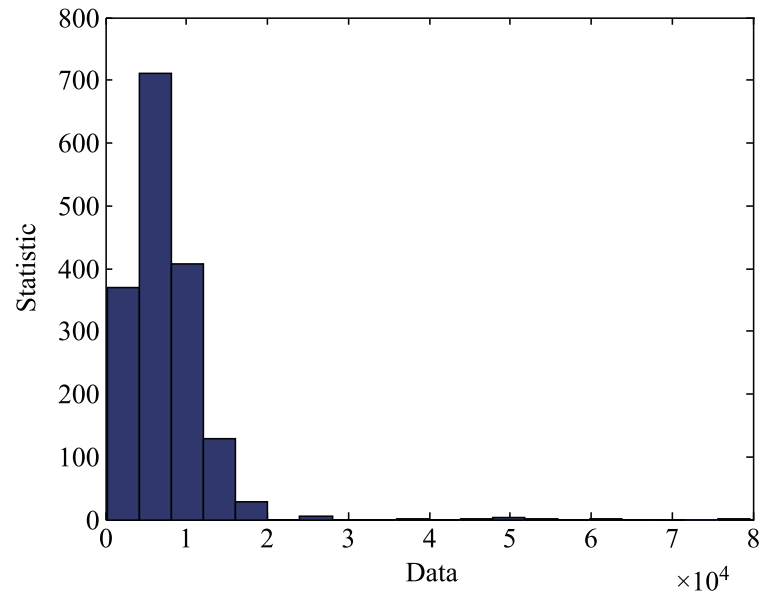

Fig. 6 Statistical histogram of target echoes $(\mathrm{SNR}=15 \mathrm{~dB})$

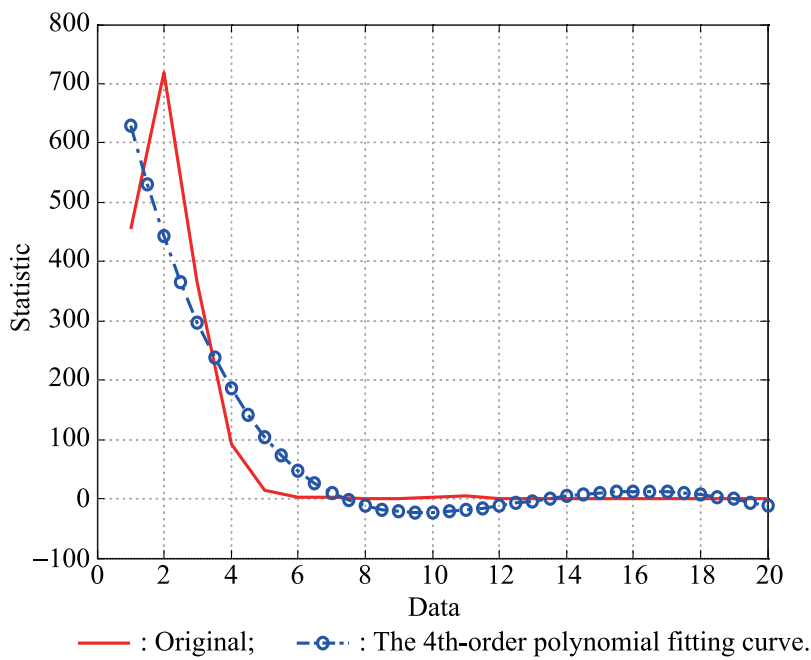

Fig. 7 Fitting curve of target echoes $(\mathrm{SNR}=15 \mathrm{~dB})$

Based on the discussion above, we can know that histogram statistical properties of target echoes are obviously different from that of noise-only echoes; histogram statistical properties of noise-only in all sliding windows are similar to each other. Hence, after the polynomial fitting of the histogram statistics curve, fitting coefficients can be exploited quantitatively to detect a range-spread target.

\subsection{Clustering analysis}

The mathematical method of classification according to certain standards is called clustering analysis, which is a kind of classification method of multi-variable statistics. The classification in both science and technology is often fuzzy, thus, the method of fuzzy clustering analysis (FCA) [38] is widely used in practice. FCA is a multi-variable technique which adopts the fuzzy mathematics method. Furthermore, based on the certain predetermined indices, FCA can accomplish classifications according to various attributes (or factors) of targets. FCA is generally divided into three parts: the data standardization, the fuzzy simila- rity matrix establishment and the clustering analysis based on the fuzzy equivalent matrix. In this paper, the method of dynamic FCA is used to detect a range-spread target. Firstly, the fitting coefficient matrix is established and then normalized; secondly, the similarity coefficient among the samples is determined by the traditional clustering method, the fuzzy similarity matrix is then built; finally, based on the fuzzy similarity matrix, the fuzzy equivalent matrix is solved by the equivalent closure method to obtain cluster analysis results. Furthermore, cluster analysis results are taken as the threshold to detect a target.

\subsubsection{Standardization}

Firstly, we define the raw data matrix $\boldsymbol{W}$. The object to be classified is called sample in FCA. Assume that the number of samples is $a . \boldsymbol{W}=\left(\boldsymbol{w}_{1}, \boldsymbol{w}_{2}, \ldots, \boldsymbol{w}_{a}\right)^{\mathrm{T}}$, each $\boldsymbol{w}_{i}$ has $b$ characteristic indices, then $\boldsymbol{w}_{i}$ can be expressed by $\boldsymbol{w}_{i}=\left(w_{i 1}, w_{i 2}, \ldots, w_{i b}\right), i=1,2, \ldots, b$. Therefore, the raw data matrix is

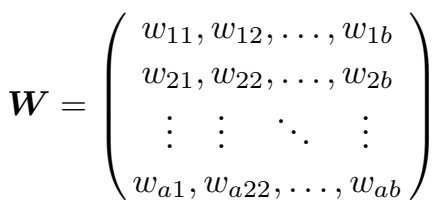

where the sample refers to the polynomial coefficients of all the sliding windows, the number of samples $a$ is the number of sliding windows, and $b$ characteristic indices correspond to polynomial coefficients with $0-4$ order. Thus the raw data matrix is the polynomial coefficient matrix in the whole sampling gates.

The polynomial coefficient matrix is then standardized. In practical applications, different data generally have different dimensions. In order to compare each variable in different dimensions, and to exclude the influence of different dimensions between variables on classification, it is necessary to standardize the raw data. Data standardization is a procedure, where the data is compressed into the range from 0 to 1 based on the requirement of the fuzzy matrix. The specific process consists of the following two parts: translation standard deviation transform and translationrange transform.

(i) Translation standard deviation transform

$$
w_{i k}^{\prime}=\frac{w_{i k}-\bar{w}_{k}}{d_{k}}, \quad i=1,2, \ldots, a ; k=1,2, \ldots, b
$$

where $\boldsymbol{w}_{i}=\left(w_{i 1}, w_{i 2}, \ldots, w_{i b}\right)$ is the sample data, the average value of $k$-dimensional data for samples with number $a$ is $\bar{w}_{k}$, standard deviation is $d_{k}$, the specific equation 
can be expressed as

$$
\left\{\begin{array}{l}
\bar{w}_{k}=\frac{1}{a} \sum_{i=1}^{a} w_{i k} \\
d_{k}=\sqrt{\frac{1}{a} \sum_{i=1}^{a}\left(w_{i k}-\bar{w}_{k}\right)^{2}} .
\end{array}\right.
$$

After the transformation, the mean value of each variable is 0 , the standard deviation is 1 , and the influence of different dimensions on variables is also eliminated, but the obtained value $w_{i k}^{\prime}$ is not necessarily in the interval $[0,1]$. Hence, the translation-range transform is required.

(ii) Translation-range transform

$$
w_{i k}^{\prime \prime}=\frac{w_{i k}^{\prime}-\min \left\{w_{i k}^{\prime}\right\}}{\max \left\{w_{i k}^{\prime}\right\}-\min \left\{w_{i k}^{\prime}\right\}}, \quad k=1,2, \ldots, b
$$

where $\max \left\{w_{i k}^{\prime}\right\}$ and $\min \left\{w_{i k}^{\prime}\right\}$ denote the maximum and the minimum among $w_{1 k}^{\prime}, w_{2 k}^{\prime}, \ldots, w_{a k}^{\prime}$. Obviously, $0 \leqslant w_{i k}^{\prime \prime} \leqslant 1$ and the influence of different dimensions is eliminated.

\subsubsection{Fuzzy similarity matrix establishment}

Secondly, based on the normalization matrix $\boldsymbol{W}$, where $\boldsymbol{W}=\left(\boldsymbol{w}_{1}, \boldsymbol{w}_{2}, \ldots, \boldsymbol{w}_{a}\right)^{\prime}, \boldsymbol{w}_{i}=\left(w_{i 1}, w_{i 2}, \ldots, w_{i b}\right)$, fuzzy similarity matrix $\boldsymbol{R}$ is established by the traditional clustering analysis method. The traditional cluster analysis method for determining the similarity degree $r_{i j}$ of $\boldsymbol{w}_{i}$ and $\boldsymbol{w}_{j}$ is mainly based on the similarity coefficient method and the distance method. In this paper, the direct Euclidean distance method is used to calculate the similarity coefficients among the samples, that is

$$
r_{i j}=d\left(\boldsymbol{w}_{i}, \boldsymbol{w}_{j}\right)=\sqrt{\sum_{k=1}^{b}\left(w_{i k}-w_{j k}\right)^{2}}
$$

\subsubsection{Fuzzy equivalent matrix establishment}

Finally, based on the fuzzy similarity matrix, the fuzzy equivalent matrix $t(\boldsymbol{R})=\boldsymbol{R}^{*}$ is provided through the equivalent closure method and then sorted on the clustering principle. In this case, clustering results can be obtained by using the truncated sets $\boldsymbol{R}_{\mu}$ obtained by different thresholds $\mu$. Hence, according to the requirement of the clustering results, the appropriate sorting threshold is selected as the test statistic to detect a distributed target.

The fuzzy similarity matrix $\boldsymbol{R}$ is an $a \times a$ square matrix. $\boldsymbol{I}$ is a unit matrix of $a$ order. If we have

$$
\left\{\begin{array}{l}
\boldsymbol{I} \leqslant \boldsymbol{R}\left(\Leftrightarrow r_{i i}=1\right) \\
\boldsymbol{R}^{\mathrm{T}}=\boldsymbol{R}\left(\Leftrightarrow r_{i j}=r_{j i}\right) \\
\boldsymbol{R} \circ \boldsymbol{R} \leqslant \boldsymbol{R}\left(\Leftrightarrow \bigvee_{k=1}^{a}\left(r_{i k} \wedge r_{k j}\right) \leqslant r_{i j}\right)
\end{array},\right.
$$

thus, it is obvious that $\boldsymbol{R}$ is a fuzzy equivalent matrix. The symbol "o" denotes composition operation of fuzzy matrices. Thus, we can utilize the transitive closure method to solve the fuzzy equivalent matrices. This algorithm mainly performs transitivity operations on similarity matrices, which makes fuzzy similarity matrices satisfy transitivity and preserve reflexivity and symmetry. In practical application, a simpler method, the square method, is generally used to solve the transitive closure $t(\boldsymbol{R})$.

Squares method: starting from the fuzzy similarity matrix $\boldsymbol{R}$, squared in turn $\boldsymbol{R} \rightarrow \boldsymbol{R}^{2} \rightarrow \boldsymbol{R}^{4} \rightarrow \ldots \boldsymbol{R}^{2^{i}} \rightarrow \ldots$ When $\boldsymbol{R} \circ \boldsymbol{R}=\boldsymbol{R}^{k}$ occurs for the first time, it indicates that $\boldsymbol{R}^{k}$ possesses transitivity, and $\boldsymbol{R}^{k}$ is the required transmission closure.

Generally, FCA should be carried out based on the classification threshold $\mu$. If $\mu$ gradually decreases, the number of classifications is smaller. Therefore, the concept of $\mu$-truncated matrix $\boldsymbol{R}_{\mu}$ of the fuzzy equivalent matrix is derived. The elements of $\boldsymbol{R}_{\mu}$ are defined as follows:

$$
\mu_{i j}=\left\{\begin{array}{ll}
1, & r_{i j}>\mu \\
0, & r_{i j}<\mu
\end{array} \quad \mu \in[0,1]\right.
$$

Obviously $\boldsymbol{R}_{\mu}$ is a Boole matrix. We can transform the fuzzy equivalence matrix into the equivalent Boole matrix based on $\boldsymbol{R}^{*}$ and $\boldsymbol{R}_{\mu}$. Therefore, $\boldsymbol{R}^{*}$ is classified by the operation on $\boldsymbol{R}_{\mu}$ and the classification results of $\boldsymbol{R}_{\mu}$ will change with $\mu(\mu \in[0,1])$. The relationship among classifications is defined by the following theorem: the matrix $\boldsymbol{R}^{*}$ is assumed to be a fuzzy equivalence matrix, $\mu_{1}, \mu_{2} \in[0,1]$ and $\mu_{1}<\mu_{2}$, the classification results determined by $\boldsymbol{R}_{\mu 2}$ is subclass determined by $\boldsymbol{R}_{\mu 1}$. Thus when the variable $\mu$ changes from 1 to 0 , the classification results determined by $\boldsymbol{R}_{\mu}$ become more detailed, and then a dynamic clustering curve is obtained. The process is called the fuzzy classification.

In this paper, the detector is designed to use the dynamic fuzzy clustering algorithm to detect targets. That is, we need to get a test statistic, which presents two distinct characteristics in the case of two kinds of data including target echoes and noise-only echoes. Therefore, it is not necessary to obtain all the classification results corresponding to threshold $\mu(\mu \in(0,1])$, and we just focus on the threshold when all data is divided into two categories. Specifically, assume that $\mu=\mu_{1}$, the classification number is called class No. 1 , when the threshold is larger than $\mu_{1}$, the classification number is called class No. 2 . That is, the target echo exists in sampling gate, the data is clustered into class No. 2. When SNR is higher, the span of the threshold becomes wide, and data in the span usually will be clustered into class No. 2 . In this case, the category is hard to change from class No. 2 to class No. 1, and the threshold 
$\mu_{1}$ required will become lower. In order to analyze the relationship between the threshold and the SNR, $10000 \mathrm{MC}$ tests are carried out under each SNR as shown in Fig. 8.

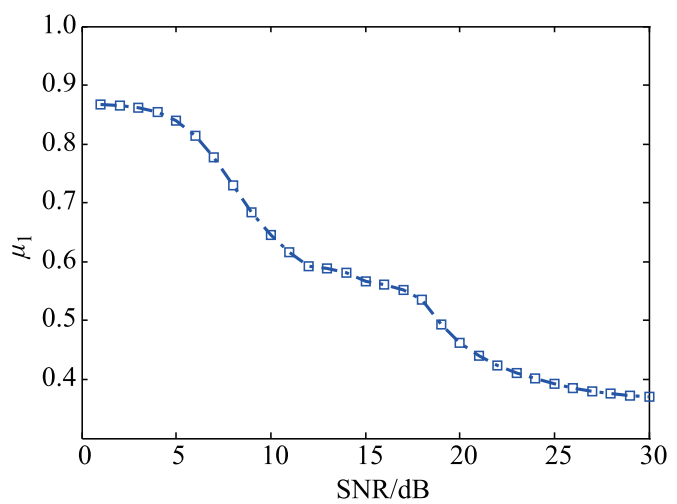

Fig. 8 Relationship between the threshold and SNR

As shown in Fig. 8, the threshold of class No. 1 is related to the SNR obviously, and when the SNR is higher, the threshold values will become lower.

\section{Performance assessment}

In this section, $\mathrm{MC}$ tests are provided to assess the performances of the detector based on echo characteristic parameters. The proposed detector is compared with other two detectors, including the scattering density dependent generalized likelihood ratio test (SDD-GLRT) detector and the energy integrating (EI) detector in white Gaussian noise. In the simulation, the target model is consistent with the model used to calculate the echo characteristic parameters, and the other parameters related to the target model are listed in Table 1. The main procedure of the proposed detector can be summarized as shown in Fig. 1. Note that the threshold $\mu_{1}$ corresponding to class No. 2 is applied as the threshold $\lambda$ of the proposed detector to detect a distributed target. In addition, the threshold $\mu_{1}$ is inversely proportional to the SNR. In this case, when the test statistic is lower than the threshold $\lambda$ calculated according to the false alarm probability $P_{\mathrm{fa}}$, then the target exists. In order to demonstrate the effectiveness and advantages of the proposed detector, numerical MC tests are given. In the following experiments, we assume that $P_{\mathrm{fa}}=1 \times 10^{-4}$, the threshold $\lambda$ is obtained from $4 \times 10^{-6} \mathrm{MC}$ tests and 10000 independent trials are conducted at each SNR level. The detection performance of the proposed detector is shown in Fig. 9.

As shown in Fig. 9, the proposed detector outperforms the SDD-GLRT detector and the EI detector. Moreover, the proposed detector still has better detection performance even at low SNR levels. In addition, in terms of the statistical complexity of the algorithm, we can know that the detector designed in this paper has the lowest complexity level. The detailed computational complexity of each detector is listed in Table 2.

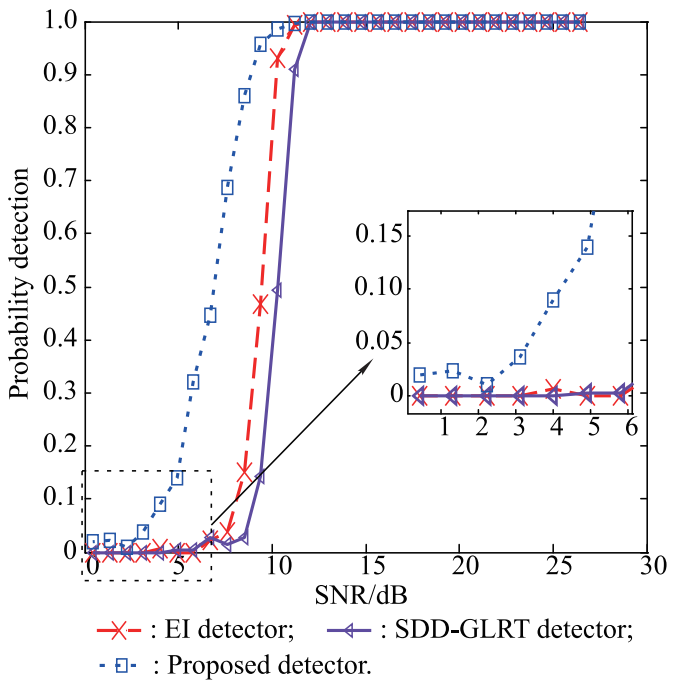

Fig. 9 Detection performance comparison

Table 2 Computational complexity of detectors

\begin{tabular}{ccc}
\hline Detector & Multiplicative times & Plus times \\
\hline EI detector & $7.8 \mathrm{e}+10$ & $1.6 \mathrm{e}+11$ \\
SDD-GLRT & $4.6 \mathrm{e}+9$ & $8.4 \mathrm{e}+9$ \\
Proposed detector & $5.0 \mathrm{e}+8$ & $3.5 \mathrm{e}+8$ \\
\hline
\end{tabular}

\section{Conclusions}

In this paper, based on different mathematical statistical characteristics of observed signals with or without targets, a detector is proposed. Firstly, the coefficient matrix of the polynomial fitting curve is calculated according to the characteristic parameters of return signals. It is found that the fitting coefficient can quantitatively reflect whether there are target echoes in the radar gate. Thus, the fitting coefficient can be utilized to represent the echo characteristics. Then, the polynomial coefficients of the statistical curves are stored as the initial matrix, and then the dynamic FCA is carried out. Finally, the threshold corresponding to class No. 1 is used as the test statistic of the proposed detector to detect a distributed target. Furthermore, the detection performance of the proposed detector is compared with that of the EI detector and the SDD-GLRT detector. The simulation result shows that the proposed detector based on echo characteristic parameters can significantly alleviate the requirement of SNR, and has a lower complexity of the algorithm.

\section{References}

[1] BOERS Y, DRIESSEN H, TORSTENSSON J, et al. Trackbefore-detect algorithm for tracking extended targets. IEE Proceedings - Radar, Sonar and Navigation, 2006, 153 (4): $345-$ 351.

[2] CONTE E, MAIO A DE. Distributed target detection in compound-Gaussian noise with Rao and Wald tests. IEEE 
Trans. on Aerospace and Electronic Systems, 2003, 39(2): $568-582$.

[3] DONG Y L, LIU M, LI K, et al. Adaptive direction detection in deterministic interference and partially homogeneous noise. IEEE Signal Processing Letters, 2017, 24(5): 599-603.

[4] ZUO L, LI M, LIU Z, et al. Range-spread target detection based on the matched ambiguity function. IET Radar, Sonar and Navigation, 2016, 10(7): $1213-1219$.

[5] XIAO L, LIU Y M, HUANG T Y, et al. Distributed target detection with partial observation. IEEE Trans. on Signal Processing, 2018, 66(6): 1551-1565.

[6] BACKMAN C G. Some recent developments in RCS measurements techniques. Proceedings of the IEEE, 1965, 53(8): $962-$ 972.

[7] LI Q, ROTHWELL E J, CHEN K M, et al. Scattering center analysis of radar targets using fitting scheme and genetic algorithm. IEEE Trans. on Antennas and Propagation, 1996, 44(2): $198-207$.

[8] MOON T T, BAWDEN P J. High resolution RCS measurements of boats. IEE Proceedings F-Radar and Signal Processing, 1991, 138(3): 218-222.

[9] AUBRY A, MAIO A DE, PALLOTTA L, et al. Radar detection of distributed targets in homogeneous interference whose inverse covariance structure is defined via unitary invariant functions. IEEE Trans. on Signal Processing, 2013, 61(20): 4949 4961.

[10] ZYWECK A, BOGNER R E. Radar target classification of commercial aircraft. IEEE Trans. on Aerospace and Electronic Systems, 1996, 32(2): 598-606.

[11] DU L, LIU H, BAO Z, et al. Radar HRRP target recognition based on higher order spectra. IEEE Trans. on Signal Processing, 2005, 53(7): 2359-2368.

[12] YANG X L, WEN G J, MA C H, et al. CFAR detection of moving range-spread target in white Gaussian noise using waveform contrast. IEEE Geoscience and Remote Sensing Letters, 2016, 13(2): $282-286$.

[13] LIU J, ZHOU S, LIU W, et al. Tunable adaptive detection in collocated MIMO radar. IEEE Trans. on Signal Processing, 2018, 66(4): 1080-1092.

[14] CAROTENUTO V, MAIO A DE, ORLANDO D, et al. Adaptive radar detection using two sets of training data. IEEE Trans. on Signal Processing, 2018, 66(7): $1791-1801$.

[15] XIAO L, LI H B, LIU Y M. Distributed target detection based on the volume cross-correlation function. IEEE Signal Processing Letters, 2018, 25(12): $1785-1789$.

[16] LIU W, LIU J, DU Q, et al. Distributed target detection in partially homogeneous environment when signal mismatch occurs. IEEE Trans. on Signal Processing, 2018, 66(14): $3918-$ 3928.

[17] WANG Z Y, LI M, CHEN H M, et al. Persymmetric detectors of distributed targets in partially homogeneous disturbance. Signal Processing, 2016, 128: $382-388$.

[18] GAO Y C, LI H B, HIMED B. Knowledge-aided range-spread target detection for distributed MIMO Radar in nonhomogeneous environments. IEEE Trans. on Signal Processing, 2017, 65(3): $617-627$.

[19] SUN S, BAJWA W U, PETROPULU A P. MIMO-MC radar: a MIMO radar approach based on matrix completion. IEEE Trans. on Aerospace and Electronic Systems, 2015, 51(3): $1839-1852$.

[20] LI B, PETROPULU, TRAPPE W. Optimum co-design for spectrum sharing between matrix completion based MIMO radars and a MIMO communication system. IEEE Trans. on Signal Processing, 2016, 64(17): 4562-4575.
[21] TANG M J, RONG Y, ZHOU J, et al. Invariant adaptive detection of range-spread targets under structured noise covariance. IEEE Trans. on Signal Processing, 2017, 65(12): 3048 - 3061.

[22] AUBRY A, MAIO A DE, et al. Radar detection of distributed targets in homogeneous interference whose inverse covariance structure is defined via unitary invariant functions. IEEE Trans. on Signal Processing, 2017, 65(3): 617-627.

[23] SHUI P, XU S, LIU H. Range-spread target detection using consecutive HRRPs. IEEE Trans. on Aerospace and Electronic Systems, 2011, 47(1): $647-665$.

[24] ZHANG X W, LI M, ZUO L, et al. A daptive subspace detection for wideband radar using sparsity in sinc basis. IEEE Geoscience and Remote Sensing Letters, 2014, 11(11): 1916 1920.

[25] LI X, CHANG W, LIU B. A new range-spread target detection algorithm for missile-borne wideband radar. Journal of Electromagnetic Waves and Applications, 2014, 28(15): $1856-$ 1868.

[26] GUO X L, TAO H H, ZHAO H Y, et al. Persymmetric rao and wald tests for adaptive detection of distributed targets in compound-Gaussian noise. IET Radar, Sonar \& Navigation, 2017, 11(3): 453-458.

[27] XU S, SHUI P, YAN X. CFAR detection of range-spread target in white Gaussian noise using waveform entropy. Electronics Letters, 2010, 46(9): 647-649.

[28] XU S, SHUI P. Range-spread target detection in white Gaussian noise via two-dimensional non-linear shrinkage map and geometric average integration. IET Radar, Sonar \& Navigation, 2012, 6(2): $90-98$.

[29] GAO Y, LI H, HIMD B. Knowledge-aided range-spread target detection for distributed MIMO radar in nonhomogeneous environments. IEEE Trans. on Signal Processing, 2017, 65(3): $617-627$.

[30] LIU J, LI H, HIMED B. Persymmetric adaptive target detection with distributed MIMO radar. IEEE Trans. on Aerospace and Electronic Systems, 2015, 51(1): $372-382$.

[31] LI H, WANG Z, LIU J, et al. Moving target detection in distributed MIMO radar on moving platforms. IEEE Journal of Selected Topics in Signal Processing, 2015, 9(8): $1524-1535$.

[32] HAO C, ORLANDO D, FOGIA G, et al. Persymmetric adaptive detection of distributed targets in partially-homogeneous environment. Digital Signal Processing, 2014, 24: $42-51$.

[33] CIUONZO D, MAIO A DE, ORLANDO D. A unifying framework for adaptive radar detection in homogeneous plus structured interference - Part I: On the maximal invariant statistic. IEEE Trans. on Signal Processing, 2016, 64(11): 2894-2906.

[34] CIUONZO D, MAIO A DE, ORLANDO D. A unifying framework for adaptive radar detection in homogeneous plus structured interference - Part II: Detectors design. IEEE Trans. on Signal Processing, 2016, 64(11): 2907-2919.

[35] MAIO A DE, ORLANDO D, HAO C P, et al. A daptive detection of point-like targets in spectrally symmetric interference. IEEE Trans. on Signal Processing, 2016, 64(12): 3207-3220.

[36] CAO S Y, ZHENG Y F, EWING R L. A wavelet-packet-based radar waveform for high resolution in range and velocity detection. IEEE Trans. on Geoscience and Remote Sensing, 2015, 53(1): 229-243.

[37] SHI B, HAO C, HOU C. Parametric rao test for multichannel adaptive detection of range-spread target in partially homogeneous environments. Signal Processing, 2015, 108: 421-429.

[38] CHAOMURILIGE, YU J, YANG M S. Analysis of parameter selection for Gustafson - Kessel fuzzy clustering using jacobian matrix. IEEE Trans. on Fuzzy Systems, 2015, 23(6): $2329-2342$. 


\section{Biographies}

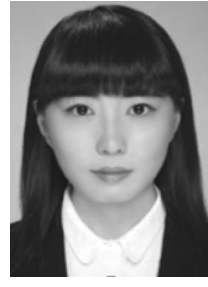

CHANG Jiayun was born in 1989. She received her master degree from Beijing Institute of Technology (BIT), China, in 2016. Currently, she is pursuing her Ph.D. degree in the School of Information and Electronics, BIT. Her research interests include automatic target recognition and radar signal processing.

E-mail: 824400828@qq.com

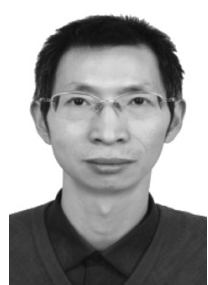

FU Xiongjun was born in 1978. He received his B.E. degree and Ph.D. degree from Beijing Institute of Technology (BIT), China, in 2000 and 2005 respectively. He is currently the vice dean of the School of Information and Electronics, Beijing Institute of Technology, and an associate professor and $\mathrm{Ph}$.D. supervisor with BIT. His current research interests include radar system, radar signal processing, waveform design, and automatic target recognition.

E-mail: fuxiongjun@bit.edu.cn

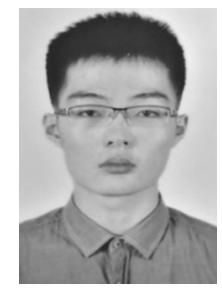

JIANG Wen was born in 1991. He received his master degree from Zhengzhou University, China, in 2016. Currently, He is pursuing his Ph.D. degree in the School of Information and Electronics, Beijing Institute of Technology (BIT). His research interests include radar signal processing and radar signal sorting.

E-mail: jwen912@126.com

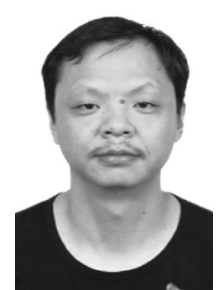

XIE Min was born in 1976. He received his Ph.D. degree from Beijing Institute of Technology (BIT), China, in 2006. Currently, his research interests include high-speed real-time signal processing, radar signal processing, and signal acquisition and storage.

E-mail: xiemin@bit.edu.cn 